\title{
Predictive Handoff Based on Mobile Positioning in CDMA System
}

\author{
Lujain S. Abdulla \\ Elect. Eng. Dept. \\ Tikrit University
}

\author{
Dr. Sami A. Mawjoud \\ Elect. Eng. Dept. \\ Mosul University
}

\begin{abstract}
In this paper, the predictive schemes of reducing call termination during handoff in Code Division Multiple Access (CDMA) system using position location in the required handoff area with minimum false handoff, where the mobile station (MSs) are located randomly in the cell and each MS has call duration, speed and direction, the speeds and directions of the MSs are varied. The signals between the MSs and Base Stations (BSs) are dealt with by using position estimation method using time difference of arrival (TDQA) of the signals, estimation of the variable MS location, knowing the speed and the direction leading to a neighboring cell. The results obtained comply with Federal Communication Commission (FCC) which is internationally adopted. Also, the blocking probabilities for handoff and for new calls using Predictive Channel Reservation (PCR) scheme and Guard Channel (GC) are studied. The results show that predictive scheme reduces the blocking probability depending on the number of reserved channels. MATLAB software (version 7.6) was used to perform the simulation programs.
\end{abstract}

Keywords: Mobile Position Estimation, Handoff CDMA System.

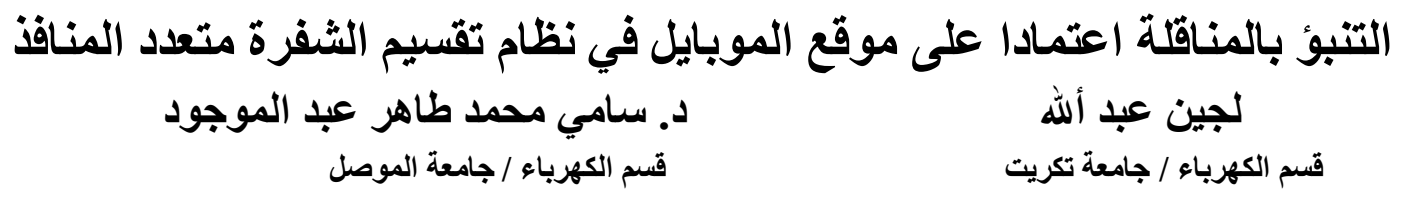

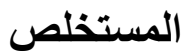

في هذا البحث تمت دراسة المخططات التتبؤية التي تقلل احتمالية قطع المكالمات أثناء عملية المناقلة في نظام

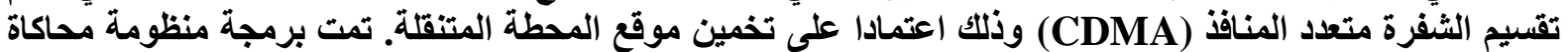

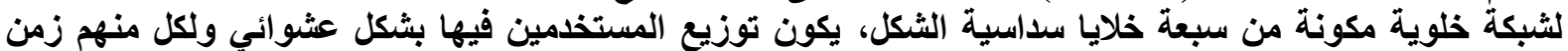

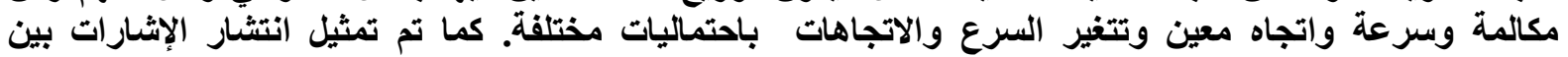

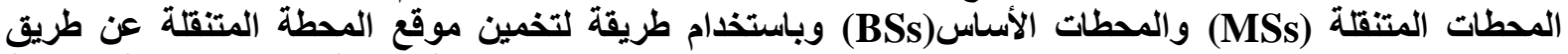

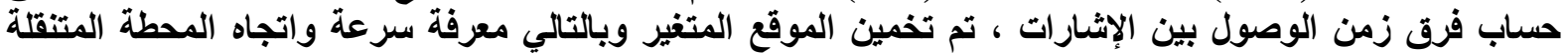

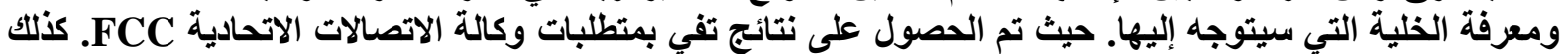

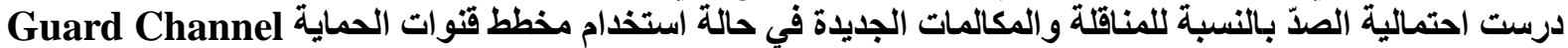

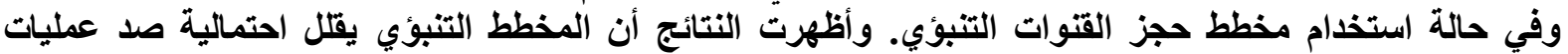

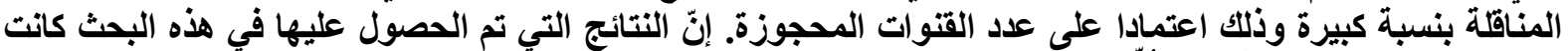

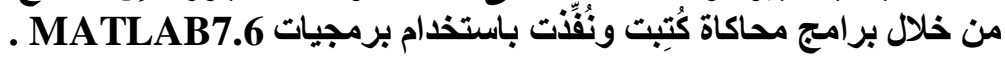

Received: 31 - 1 - 2011

Accepted: 5 - 7 - 2011 


\section{Introduction:}

The U.S. Federal Communication Commission (FCC) has made E911 a mandatory requirement for wireless communication services such as cellular telephone, wideband (broadband) personal communication and geographic area Specialized Mobile Radio (SMR). This ruling and upcoming service is called wireless E911. The FCC requires that Public Safety Answering point (PSAP) attendants of wireless communication network must be able to know a 911 user's phone numbers for return calls and the location of the user, so that calls can be routed to appropriate PSAP and related emergency assistance attendants [1].

In the year 2000, the FCC declared whether they will offer a network based or handset based solution to the position determination.

For offering network based solution or handset-based solution, the position accuracy [2] is as in table (1).

Table (1) Accuracy Required for Locating Mobile Phones [2]

\begin{tabular}{|c|c|c|}
\hline Solutions & $67 \%$ of calls & $95 \%$ of calls \\
\hline Handset-Based & 50 meters & 150 meters \\
\hline Network-Based & 100 meters & 300 meters \\
\hline
\end{tabular}

\section{Position Location Methods:}

There are a number of different radio frequency base techniques [2] that can be used for wireless Position Location (PL) such as cell identify cell 1D, Ground Positioning System GPS. Assisted GPS, (AGPS). Time of Arrival (TOA) and Time Difference of Arrival (TDOA). The issue is how effectively and efficiently a technique that can be implemented an incorporated in the existing mobile system without requiring changes either at the user (MS) end or at the service provider (BS) end.

The TDOA positioning estimation is one of the most popular method within Cellular Localization. The TDOA position location is accomplished in two stages. The first stage involves estimation of the TDOA between receivers through the use of time delay estimation techniques. The estimated TDOAs are then transformed into range difference measurements between three base stations resulting in a set on non-linear hyperbolic range difference equations. The second stage utilizes efficient algorithm to produce an unambiguous solution to these nonlinear hyperbolic equations. The solution obtained by these algorithms results in the estimated position location of the source. This method is sometimes called a hyperbolic position location [3][4].

\section{Theoretical Analysis:}

\subsection{TDOA Estimation:}

The TDOA of a signal can be estimated by cross-correlation techniques, in which the received signal at one base station is correlated with the received signal at another base station [3].

For a signal $\mathrm{s}(\mathrm{t})$ radiating from a remote source through interference and noisy channel, a model for the time delay estimation between the received signal at two base stations, $\boldsymbol{x}_{1}(t)$ and $\boldsymbol{x}_{2}(\boldsymbol{t})$, is given by [5]

$x_{1}(t)=A_{1} s\left(t-d_{1}\right)+n_{1}(t)$

$x_{2}(t)=A_{2} s\left(t-d_{2}\right)+n_{2}(t)$

Where:

$A_{1}$ and $A_{2}$ are the amplitude scaling of the signals.

$\mathrm{n}_{1}(\mathrm{t})$ and $\mathrm{n}_{2}(\mathrm{t})$ consist of Gaussian noise and interfering signals . 
$\mathrm{d}_{1}$ and $\mathrm{d}_{2}$ are the signal delay times.

This model assumes that $\mathrm{s}(\mathrm{t}), \mathrm{n}_{1}(\mathrm{t})$ and $\mathrm{n}_{2}(\mathrm{t})$ are real and jointly stationary, zero mean (time average) random processes and that $s(t)$ is uncorrelated with noise $n_{1}(t)$ and $n_{2}(t)$. Referring to the delay time and scaling amplitudes to the receiver with the shortest time of arrival, assuming $d_{1}<d_{2}$, the model of (1) can be rewritten as[5]

$x_{1}(t)=s(t)+n_{1}(t)$

$x_{2}(t)=A s(t-D)+n_{2}(t)$

Where:

A is the amplitude ratio.

$\mathrm{D}=\mathrm{d}_{2}-\mathrm{d}_{1}$.

It is desired to estimate $\mathrm{D}$, the time difference of arrival (TDOA) of $\mathrm{s}(\mathrm{t})$ between the two receivers. It may also be desirable to estimate the scaling amplitude A.

\subsection{Chan's Method}

Following Chan's method, for a three base station systems $(\mathrm{M}=3)$, producing two TDOA's, $x$ and $y$ can be solved in terms of where $\mathrm{R}_{1}$ is the range of the first BS station which is assumed to control the call and the first to receive the transmitted signal. The solution is in the form of [3]

\section{Practical Methods for Solving Hyperbolic Equations:}

The methods that can be practically used for solving hyperbolic equations are Taylorseries method, Fang's method and Chan's method .Among these, Fang's and Chan's methods provide a closed form exact solution which is not available with Taylor-series method and also are computationally less intensive [4].

$$
\left(\begin{array}{l}
x \\
y
\end{array}\right)=-\left[\begin{array}{ll}
X_{2,1} & Y_{2,1} \\
X_{3,1} & Y_{3,1}
\end{array}\right]^{-1} *\left\{\left(\begin{array}{l}
R_{2,1} \\
R_{3,1}
\end{array}\right) R_{1}+\frac{1}{2}\left(\begin{array}{c}
R_{2,1}^{2}-K_{2}+K_{1} \\
R_{3,1}^{2}-K_{3}+K_{1}
\end{array}\right)\right\}
$$

Where:

$K_{1}=X_{I}^{2}+Y_{1}^{2}$

$K_{2}=X_{2}^{2}+Y_{2}^{2}$

$K_{3}=X_{3}^{2}+Y_{3}^{2}$

A general model for two dimension (2D) position location estimation at a source using $\mathrm{M}$ base stations is developed. Referring all TDOAs to the first BS which is assumed to be the base station controlling and the first to receive the transmitted signal.

Let the number of BS index $\mathrm{i}=2, \ldots, \mathrm{M}, \mathrm{X}$ and $\mathrm{Y}$ be the source location and $\mathrm{X}_{\mathrm{i}}, \mathrm{Y}_{\mathrm{i}}$ be the known location of the receiver. The square range distance between the source and the $i^{\text {th }}$ receiver is given as in [3].

$$
\begin{aligned}
R_{i} & =\sqrt{\left(X_{i}-x\right)^{2}+\left(Y_{i}-y\right)^{2}} \\
& =\sqrt{X_{i}^{2}+Y_{i}^{2}-2 X_{i} x-2 Y_{i} y+x^{2}+y^{2}}
\end{aligned}
$$

When equation (3) is inserted into equation (4), with (the number at BS index) $i=1$, a quadratic equation in terms of $R_{1}$ is produced. 
Substituting the positive root back into (3) results in the final solution. Two positive roots may exist from the quadratic equation that can produce two different solutions, resulting in an ambiguity. This problem can be resolved by using priori information [3].

When the quadratic equation in $\mathrm{R}_{1}$ is obtained in the form[4]

$a R_{1}^{2}+b R_{1}+c=0$

only the following root should be considered for cellular position location.

$R_{1}=\frac{-b-\sqrt{b^{2}-4 a c}}{2 a}$

To evaluate the accuracy of the hyperbolic position location technique, a commonly used measure of PL accuracy is the Root Mean Square (RMS). The RMS position location error is as in[5]

$E_{R M S}=\sqrt{\varepsilon}=\sqrt{E\left[(x-\hat{x})^{2}+(y-\hat{y})^{2}\right]}$

Where:

$\hat{x}$ and $\hat{y}$ are the estimated values of $\mathrm{x}$ and y coordinate of MS position location.

\subsection{Generalized Cross-Correlation Methods:}

For accurate estimation of time difference of arrival D requires the use of estimation techniques which is immute to noise and interference. The ability to resolve multipath signal components, generalized cross correlation (GCC) method is used. GCC method crosscorrelate pre-filtered versions of the received signals at the two receiving stations, then estimate the TDOA between them as the location of the peak of the cross correlation estimation. Pre-filtering is intended to accentuate frequencies for which signal-to-noise (SNR) is highest and to attenuate the noise before the signal is passed to the correlator [5].

When $\boldsymbol{x}_{1}(t)$ and $\boldsymbol{x}_{2}(t)$ are filtered, the cross power spectral density between the filtered outputs is given by[6]

$$
G_{y 2 y 1}(f)=H_{1}(f) H_{2}^{*}(f) G_{x 2 x 1}(f)
$$

Where:

* denotes the complex conjugate. Therefore, the generalized cross-correlation, specified by superscript $\mathrm{G}$, between $\boldsymbol{x}_{1}(\boldsymbol{t})$ and $\boldsymbol{x}_{2}(\boldsymbol{t})$ is

$R_{y 2 y 1}^{G}(\tau)=\int_{-\infty}^{\infty} \Psi_{G}(f) G_{x 2 x 1}(f) e^{j \pi f \tau} d f$

Where

$\Psi_{G}(f)=H_{1}(f) H_{2}^{*}(f)$

and denotes the general frequency weighting, or filter function. Because orlv an actimate of $\boldsymbol{R}_{y 2 y 1}^{G}(\boldsymbol{\tau})$ can be obtained, equation (9) is rewritten as [4]

$\widehat{R}_{y 2 y 1}^{G}(\tau)=\int_{-\infty}^{\infty} \Psi_{G}(f) \hat{G}_{x 2 x 1}(f) e^{j \pi f \tau} d f$

Which is used to estimate $\mathrm{D}$. The GCC methods use filter functions $\Psi_{\mathrm{G}}(\mathrm{f})$ to minimize the effect of noise and interference [6] as shown in figure (1). 


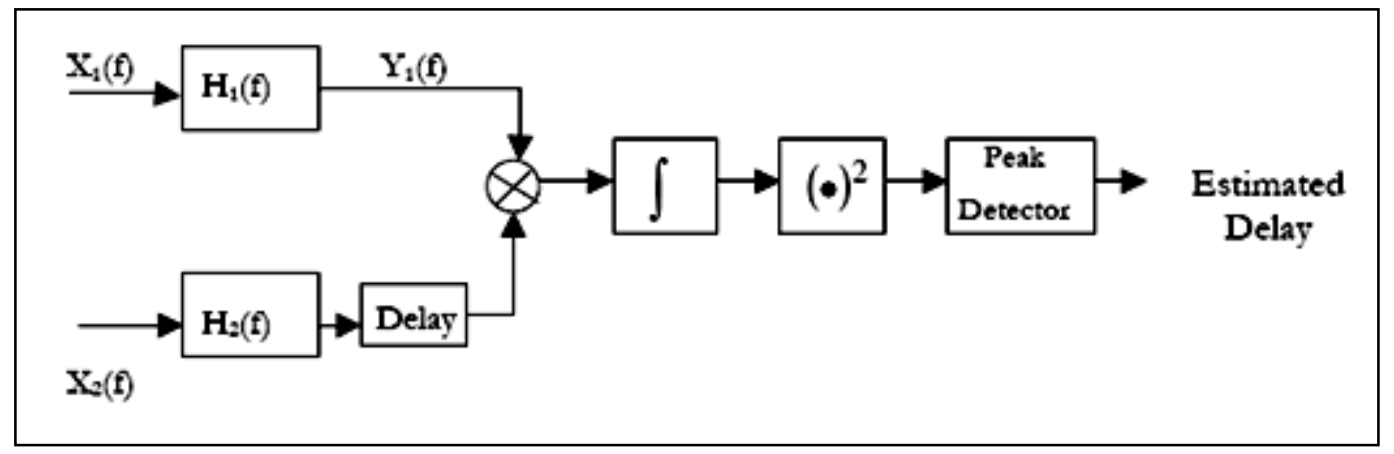

Fig. (1) Generalized cross-correlation method for TDOA estimation [7]

\section{Predictive Schemes for Handoff:}

Handoff in cellular networks is the mechanism that transfers an ongoing call from the current cell to the next cell as the mobile station (MS) moves through the coverage area of the cellular system. A successful handoff provides continuation of the call which is vital for the perceived Quality of Service (QoS). Since, the blocking of a handoff request is less desirable than the blocking of a new call. Specific schemes have been proposed to prioritize handoff requests, e.g., channel reservation and handoff queuing. The Guard Channel (GC) protocol is the most popular non predictive channel reservation approach for handoff prioritization. The GC-based scheme improves the acceptance rate of handoffs, but could greatly decrease the total traffic of the cellular network due to the increased blocking of new calls [9][10].

Predictive Channel Reservation (PCR) schemes have also been presented by [10] and also used in [8][9] [11]. These schemes have primarily focused on using probabilistic predictions of the MS movement.

\section{Predictive Channel Reservation (PCR):}

In PCR scheme, each MS periodically measures its position and orientation. The position measurement is made by using TDOA and the information is sent to the BS through uplink message .The BS uses this information to predict the projected future path of the MS. Based on the projected path, the next cell (one of the neighboring cells of the current cell) that the mobile is heading to is computed. When the mobile is within a certain distance from the next cell, the current base station makes a reservation request to the new base station in order to pre-allocate a channel for the expected handoff event. A reservation may be deemed invalid (false reservation) at a later time .In this case, cancellation of reservation must be sent to

re-allocate the reserved channel [8][10].

In PCR there are two types of cancellations that are likely to occur, they are motion cancellation and termination cancellation as explained below [8][9].

- Motion Cancellation: Cancellations in this category occur when the mobile deviates from its predicted path and starts heading to a neighboring cell different from the one that was computed in the latest prediction. The frequency of occurrence of motion cancellations depends primarily on the mobility pattern of the MS and the length of the interval between two successive position measurements.

- Termination Cancellation: This type of cancellation occurs when the ongoing call terminates before the mobile reaches the new cell boundary. In this case, a cancellation is sent to the new BS to de-allocate the channel reserved for this MS.

The reservation and cancellation mechanism is illustrated in figure (2). 
Figure (2) shows a small portion of a cellular network containing three overlapping cells. Handoff occurs when the mobile reaches the boundary of the current cell .In this figure, mobile $\mathrm{V}_{1}$ is ready to handoff initiation from cell $\mathrm{A}$ to cell $\mathrm{B}$, and will continue its call if cell $\mathrm{B}$ has an available channel. The following example of motion cancellation is illustrated in figure (2). At time $t_{1}, B_{A}$ sends to $B_{B}$ a reservation request for mobile $\mathrm{V}_{2}$. At time $\mathrm{t}_{2}, \mathrm{BS}_{\mathrm{A}}$ sends to $\mathrm{BS}_{\mathrm{B}}$ a cancellation for $V_{2}$ and sends to $B S_{C}$ a reservation for $\mathrm{V}_{2}$ (after realizing that this mobile is heading to $\mathrm{BS}_{\mathrm{C}}$ rather than $\mathrm{BS}_{\mathrm{B}}$ ). The length of the duration of the false reservation in cell $B S_{B}$ is equal to $t_{2}-t_{1}$ [8][9].

Base stations in the predictive scheme

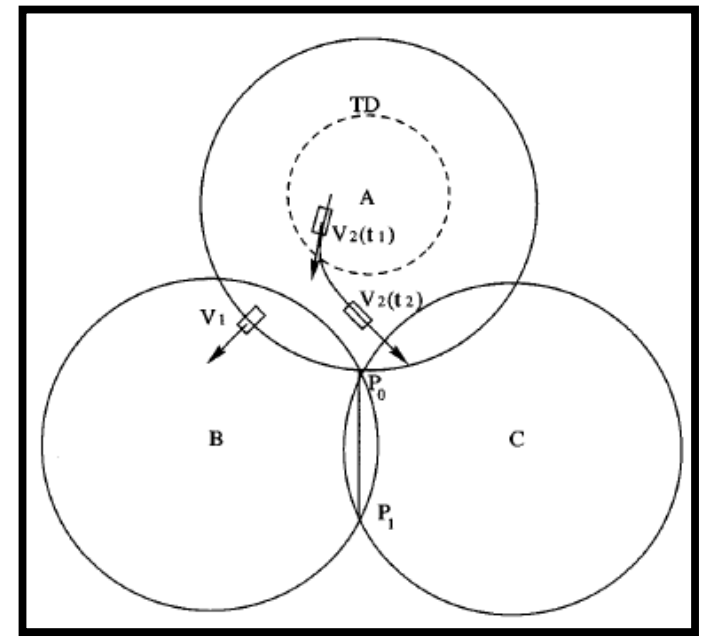

Fig. (2) Reservation and cancellation request [8] handle four types of control messages related to channel allocation. Below is a brief description of how the base station handles each request [10].

- Handoff Request :is dropped only if there is no free channel or reserved channel that can be used for this handoff. A concept called reservation pooling is used to enhance the benefits of the predictive scheme.

- New Call Request: is dropped if there is no free channel that can be used for this new call.

- Reservation Request: is ignored if there is no free channel that can be converted into a reserved channel.

- Cancellation Notification: causes a reserved channel to be converted to a free channel.

\section{- Reservation Pooling:}

Rather than strictly mapping, each reserved channel to the mobile that made the reservation, the set of reserved channels at any moment is used as a generic pool to serve any handoff request. If there is no free channel, a reserved channel may then be used for a handoff request that does not have prior reservation [8][9][10].

\section{- Selection of the Threshold Distance:}

The concept of Threshold Distance (TD) is used to control the timing of reservation submission; and reduces the occurrences of early reservations. TD is defined as a distance smaller than the radius of the cell and therefore specifies an inner circle inside the (circular) cell. If the mobile is currently located inside the TD circle, the base station sends no reservation. The value of TD needs to be carefully selected: large values of TD may cause undesirable delay in the submission of true reservations; small values of TD may cause frequent false reservations [8][10].

\section{Simulation and Results:}

\section{Mobile Positioning by Using TDOA Model}

The system used in this simulation is IS-95 CDMA. TDOA method does not require any information from the downlink; therefore, the main concern is the uplink. Most of the parameters used comply with the IS-95 CDMA cellular standard. The BS configuration used for the simulation is based on a hexagonal cellular layout with seven cells cluster as shown in figure (3). Table (2) lists the parameter used in TDOA simulation. 
- General Structure of the Simulations:

In this section, a description of the simulation programs will be presented as follows[4]:

- Random binary data are generated for different users and spread by using spreading codes (Gold code and Kasami code), then sampling this data. The length of the data is set according to the desired length of the snap shot (12 bit) for TDOA PL .This is done for all users in all the cells.

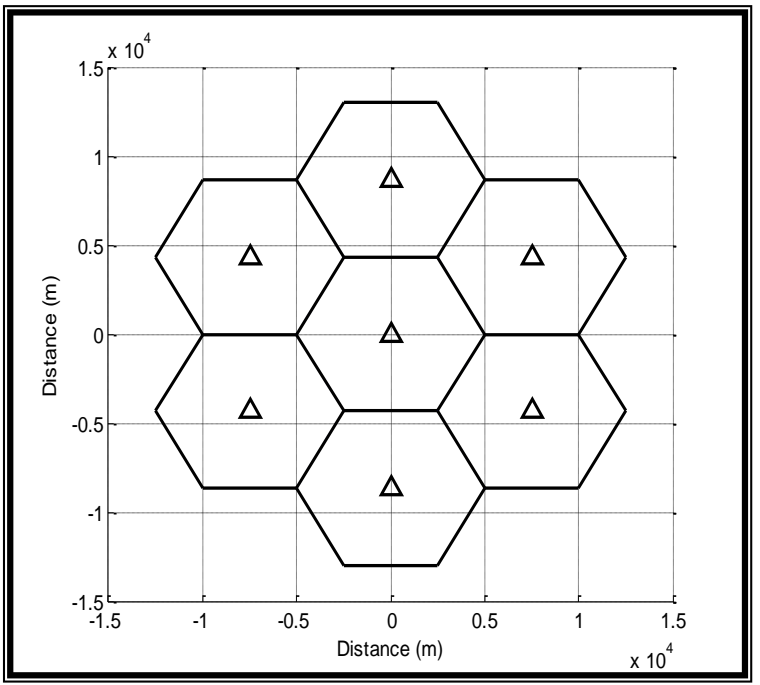

Fig. (3) Seven cells cluster

Table (2) Parameters used in TDOA simulation

\begin{tabular}{|c|c|}
\hline Parameter & Value \\
\hline Number of cells & 7 \\
\hline Cell radius (R) & $5000 \mathrm{~m}$ \\
\hline Number of users in the cell & 20 \\
\hline Bandwidth & $12288 \mathrm{M}$ chip per sec. \\
\hline Snap shot & 16 sample per chip \\
\hline Sample rate & 128 \\
\hline Processing gain & 4 \\
\hline $\mathrm{E}_{\mathrm{b}} / \mathrm{N}_{\mathrm{o}}$ & $4 \mathrm{~dB}$ \\
\hline $\begin{array}{c}\text { Number of rake receiver } \\
\text { fingers }\end{array}$ & \\
\hline
\end{tabular}

- Calculating the signal delays and attenuations experienced by each user's signal for each BS, depending on the distance and the path loss model being used.

- The signals are subjected to a Rayleigh fading effect.

- Additive White Gaussian Noise (AWGN) is added to the signals depending on the desired $E_{b} / N_{o}(10 \mathrm{~dB})$. The flow chart of the received signal for the desired user from one path is shown in figure (4), usually the signal of a user received by the BS is multipath.

- The received signals, at each base station, are incoherently added from all the users, as shown in figure (5).

- After all the received signals have been computed, the process of identifying the data of the desired user begins. Since, the signal is very weak at the neighboring base stations, interference cancellation is used at the base stations in some of the simulations discussed for comparison, in order to access the improvement obtained. The neighboring cells measure the desired MS signal by subtracting (by cross- 
correlation) the MS's signals in this cell, in order to obtain stronger signal from the desired user.

- The data bits for the desired user are despread to form the snap shot so that it contains only the desired user's spread signal.

- The cross-correlation has done to get the hyperbolic equations.

- Chan's algorithm is used to solve the hyperbolic equation to get the PL estimate of the desired user. This process is shown in figure (6), the controlling base station is referred to as $\mathrm{BS}_{1}$ and the other two as $\mathrm{BS}_{2}$ and $\mathrm{BS}_{3}$.

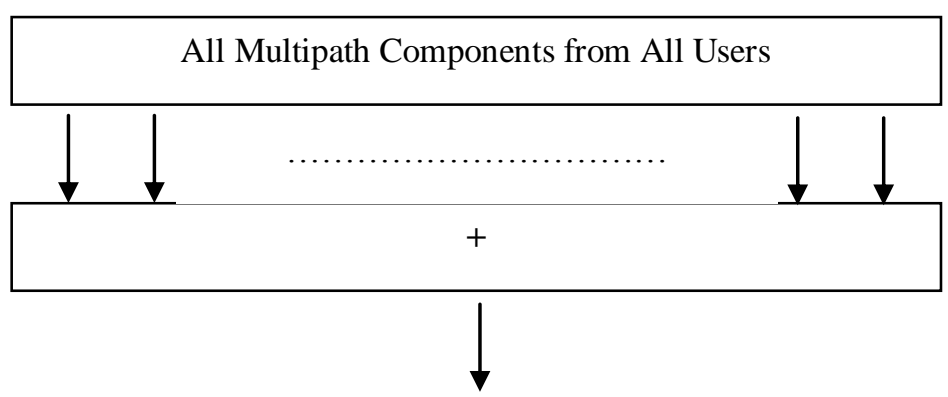

Received signal at a Base Station

Fig. (5) Composition of received signal at base station [4]

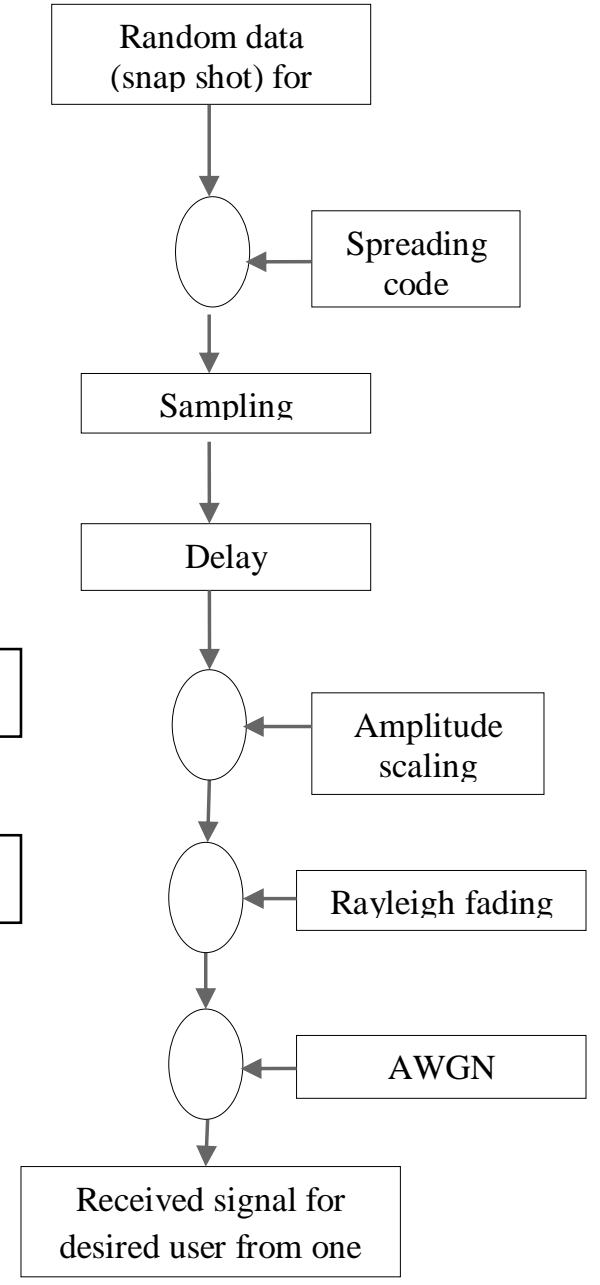

Fig. (4) Simulation flow chart for the signal of a single user

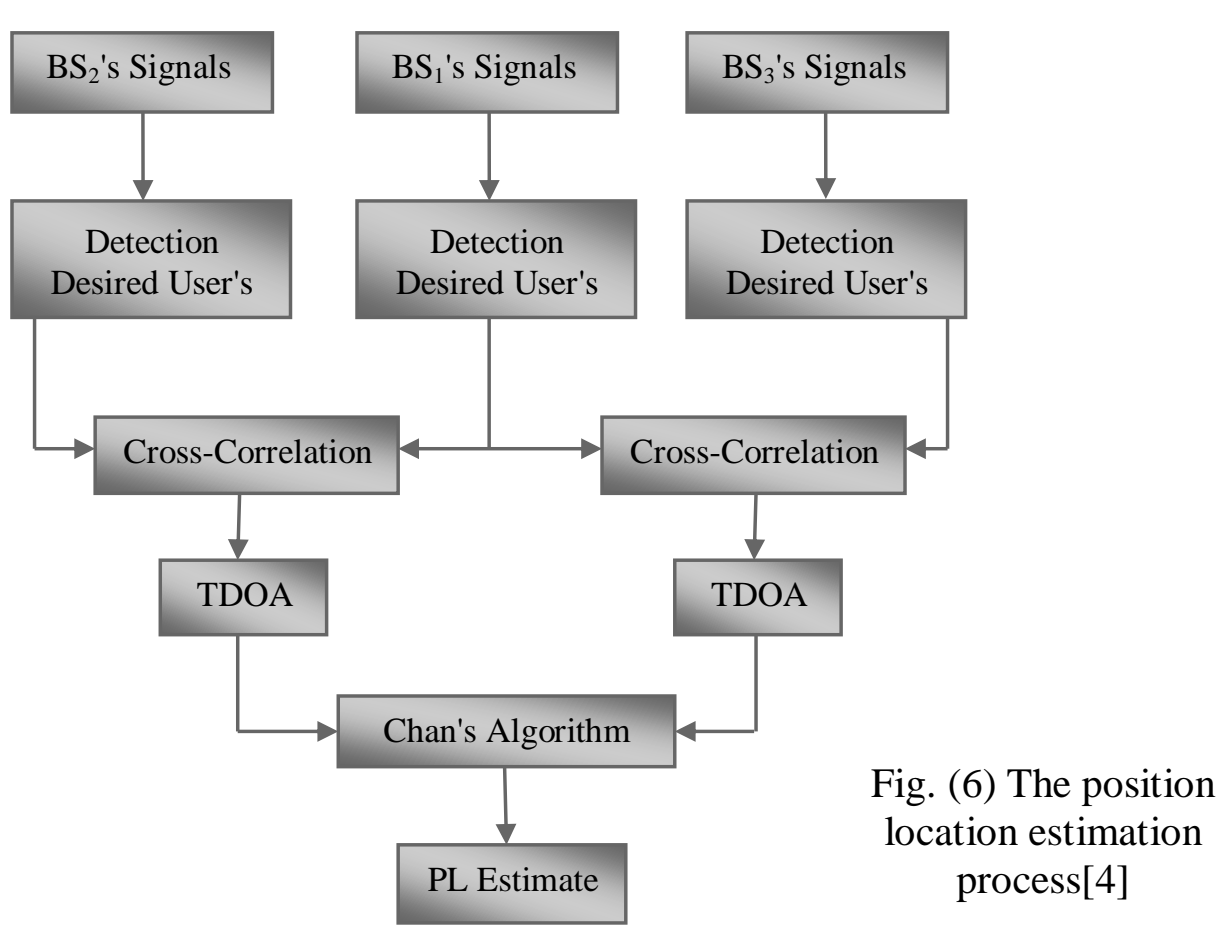

「4] 


\section{- Interference Cancellation}

The challenging problem in position location using TDOA is to obtain a good signal for the required user in neighboring cells. One feasible method to solve this problem is to use multiuser receiver that has the ability to reduce the Multiple Access Interference (MAI) to increase the SNR of the required signal at those base stations. The effect of using or not using the interference cancellation at the neighboring base stations has been discussed in the next section. In this simulation, one stage of parallel interference cancellation has been used to reduce MAI for the desired user's signal at the base stations other than the controlling BS. An expression, that approximately gives the optimal value for this Interference Reduction Factor (IRF), depending on the number of users and the processing gain is[4]:

IRF $=0.95-0.48(\mathrm{k} / \mathrm{N})$

Where:

$\mathrm{k}$ is the number of users.

$\mathrm{N}$ is the processing gain of the CDMA system.

In the simulations, up to 20 users in each cell are assumed and the IS-95 processing gain is 128 . Hence, 0.875 for the interference reduction factor has been obtained.

\section{- Effect of Interference Cancellation on Average Error:}

In this simulation, TDOA estimation calculated in the center cell $\left(\mathrm{BS}_{1}\right)$ as in figure (3), while the signals from all MSs in other cells interfere with the MSs in this cell. In TDOA, the mobile signals measured by the three $\mathrm{BSs}$ and followed by cross-correlation between them to estimate $\mathrm{TDOA}_{12}$ and $\mathrm{TDOA}_{13}$. In the $\mathrm{BS}_{1}$, there is no problem concerning the signal of the desired MS to be located, but if the MS is near $\mathrm{BS}_{1}$, the signal received by $\mathrm{BS}_{2}$ and $\mathrm{BS}_{3}$ will be weak due to path loss, also due to the MSs in $\mathrm{BS}_{2}$ and $\mathrm{BS}_{3}$ and this will increase the error in MS position estimation. To avoid the problems mentioned above is by:

- Increasing the power of mobile at the estimated time only, the power control sends the order to this mobile to increase its power at this time. Figure (7) shows the effect of power increasing on average error.

- Using interference cancellation to decrease the required power at the estimated time (by increase signal to noise ratio). Figure (7) shows the effect of interference cancellation method and its advantage over increasing the power only. The power needed when interference cancellation is used is $3.5 \mathrm{~dB}$ compared to $6.3 \mathrm{~dB}$ with no interference cancellation.

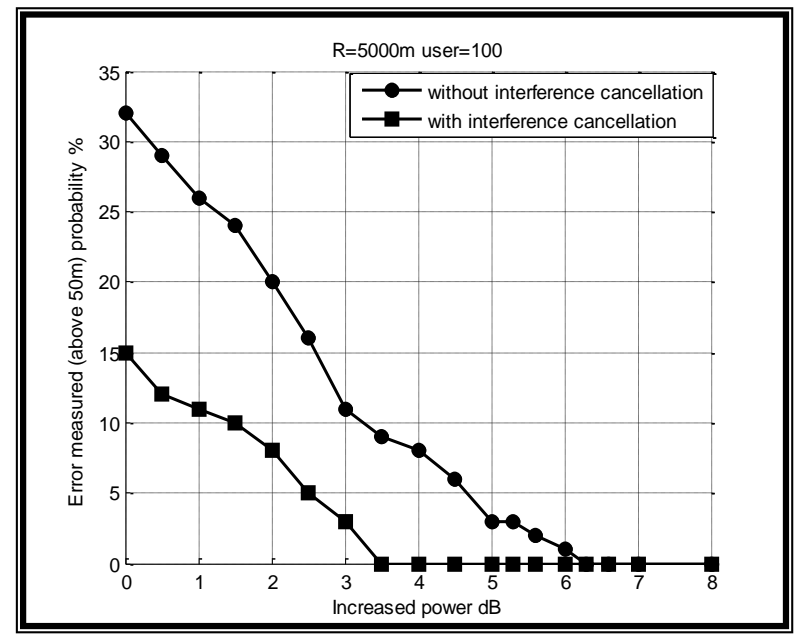

Fig. (7) Error probability with increased power

Figure (7) shows the percentage of the of error measured estimating (above $50 \mathrm{~m}$ or $1 \%$ error/R) with and without interference cancellation method.

Note that, the interference cancellation method decreased the error from $32 \%$ to $15 \%$ without an increase in power. 


\section{CDMA System Model and Soft Handoff:}

\subsection{Simulation Models and Parameters:}

The CDMA system used in the simulation is a 7 cell cluster, the MSs in these cells are randomly (uniformly) distributed. At the beginning of the simulation, each MS has initial location, speed and direction with random distribution. The number of MSs is between one and twenty in each cell. The time of calls for all users are distributed exponentially with a holding time $180 \mathrm{sec}$ (average time for one call). The number of calls in the simulation depends on the traffic load (the utilization time of all channels in a cell). Where:

Traffic load $=($ new call arrived $*$ holding time $) /$ number of channels.

The parameters used in the simulation [11] are shown in table (3).

The MSs move with speeds between $0 \mathrm{~m} / \mathrm{sec}$ and $20 \mathrm{~m} / \mathrm{sec}$ with random distribution.

Two models for mobiles movement are used in simulation:

Model I: not all users change their speeds and directions.

Model II: users change their speeds and directions with different probability for special angles $\left(45^{\circ}\right.$ and $\left.90^{\circ}\right)$. This change in direction is updated periodically every $60 \mathrm{sec}$. The probability of directions changing and the angles are shown in table (4) [11].

Tble (3) Parameters used in handoff simulation

\begin{tabular}{|c|c|}
\hline Value & Parameter \\
\hline 7 & Number of cells \\
\hline 20 & Number of channels in a cell \\
\hline $180 \mathrm{sec}$. & Holding time \\
\hline$[0-20] \mathrm{m} / \mathrm{sec}$ & Speed \\
\hline $12 \mathrm{~h}$ & Time of simulation \\
\hline 50 or 25 & Number of simulation runs \\
\hline $10 \mathrm{sec}$. & Period time of measurement \\
\hline $80 \%$ & Traffic load \\
\hline $15 \%$ & Soft handoff overhead \\
\hline $65 \%$ & Threshold distance TD \\
\hline
\end{tabular}

Table (4) Probability of directions changing [11]

\begin{tabular}{|c|c|}
\hline Value & Parameter \\
\hline $10 \%$ & $\begin{array}{c}\text { Probability with which a } \\
\text { MS stops }\end{array}$ \\
\hline $63 \%$ & $\begin{array}{c}\text { Probability of keeping } \\
\text { original moving direction }\end{array}$ \\
\hline $9 \%$ & $\begin{array}{c}\text { Probability of making a } 45^{\circ} \\
\text { turn }\end{array}$ \\
\hline $18 \%$ & $\begin{array}{c}\text { Probability of making a } 90^{\circ} \\
\text { turn }\end{array}$ \\
\hline
\end{tabular}

\subsection{Variation of Traffic Load Versus New Call Blocking Probability and Soft Handoff Blocking Probability:}

The new calls begin at random time. A new call is established if there is a free channel in the cell, else the call is blocked. When a MS arrives at the handoff region, a free channel from the new cell (the MS moving to) is allocated, else the handoff will be blocked.

Figures (8) and (9) show the relation between the blocking probability (for new call and soft handoff ) with traffic load for model I and model II at soft handoff overhead $15 \%$ (the value of soft handoff region over the entire cell area).

The new call blocking probability is greater than the handoff blocking probability because the handoff has priority on a new call initiation.

Figure (10) shows the relation between false handoff probability, (if the user is allocated a channel for the handoff then complete its call before crossing the handoff region) in that cell and traffic load, while figure (11) shows the relation between the average number 
of soft handoffs and traffic load, where the number of handoffs is increased with an increase of the traffic load.

The blocking probability and false handoff probability in model II are greater than of model I, but the average number of handoffs is less because of the change of directions for some MSs in model II.

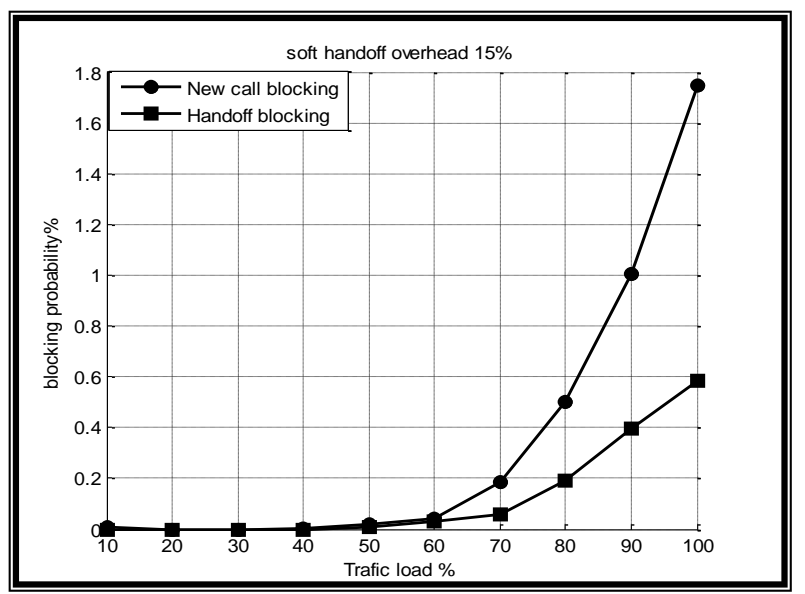

Figure (8) Blocking probability for model I

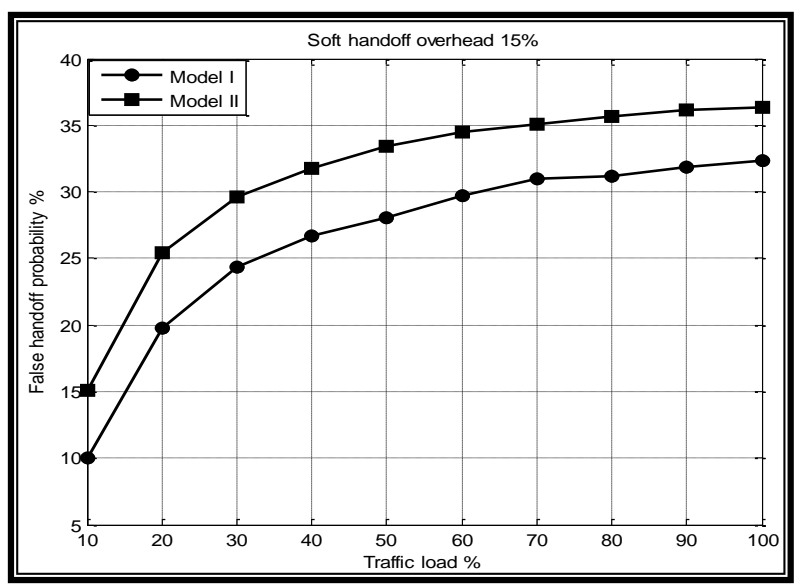

Figure (10) False handoff probability

\subsection{Predictive Schemes of Soft Handoff:}

The important type of predictive handoff is the predictive based on the mobile positioning. Predictive Channel Reservation (PCR) method is a predictive handoff method depending on mobile positioning, therefore, the results obtained by simulations of this method will be discussed.

Figure (12) illustrates the cells with the soft handoff regions and the threshold distance (TD) for PCR method.

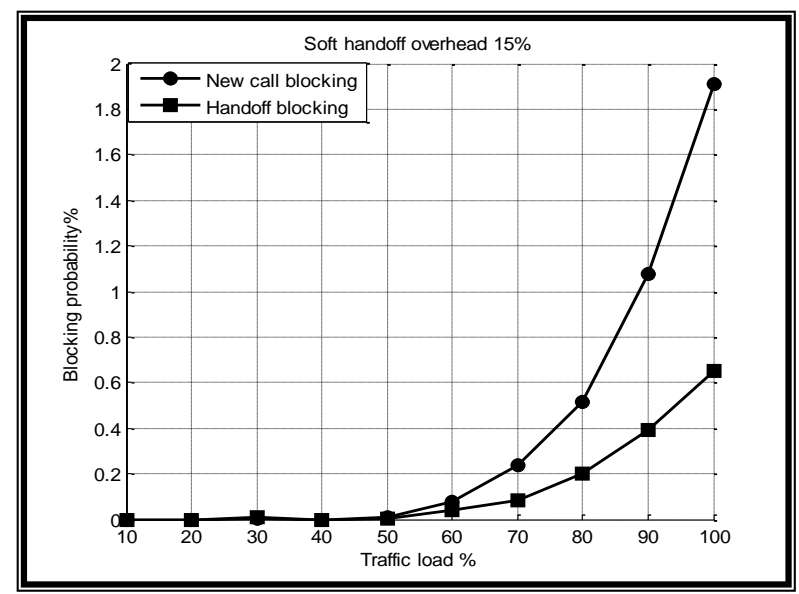

Figure (9) Blocking probability for model II

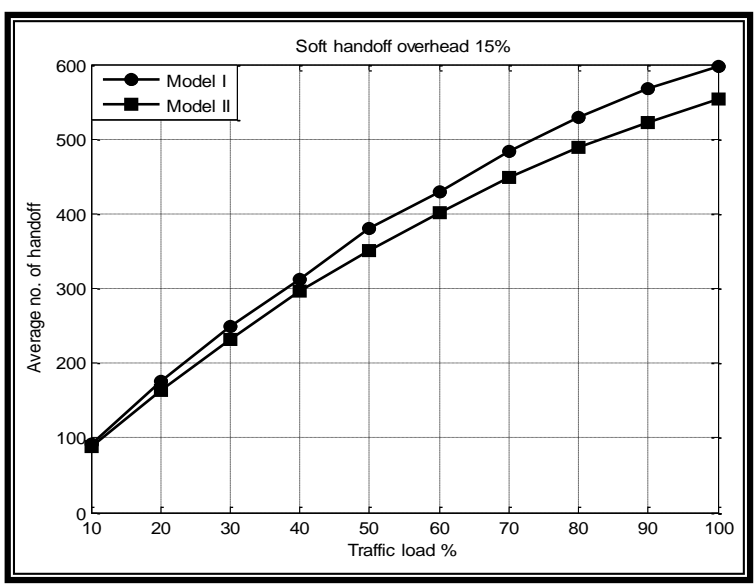

Figure (11) Average number of handoffs

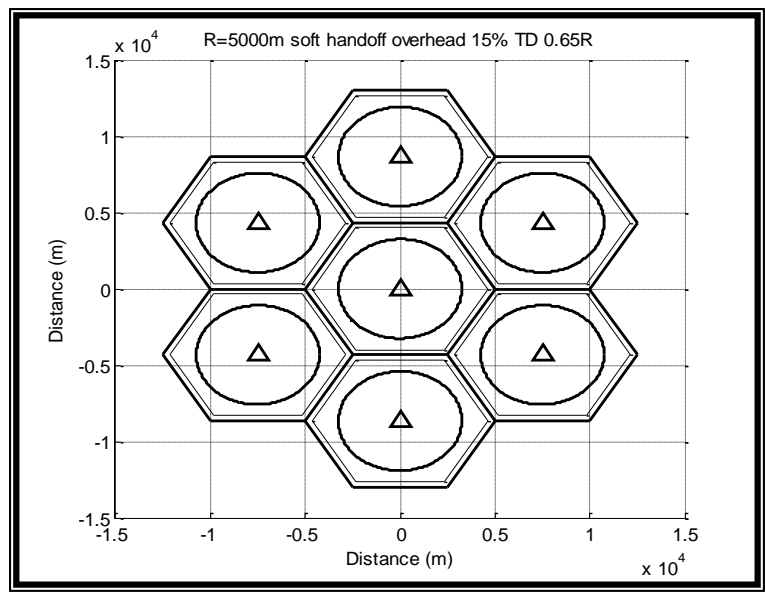

Fig. (12) Soft handoff and TD regions 


\subsection{PCR with Reservation Pooling:}

Figure (13) shows the relation between the handoff blocking probability with the number of reserved channels for model I and model II. The increase in the number of the reserved channels will decrease the handoff blocking probability, while the new call blocking probability will increase with increasing number of reserved channels as is shown in figure (14) for model I and model II. The reason of decreasing the handoff blocking probability in figure (13) is that when a MS arrives at handoff region, it is allocated a channel for handoff from reservation pooling if such channel is available (when the number of reserved channels increases the number of channels in reservation pooling will increase), increasing the number of the reserved channels causes decrease in free channels hence increasing the new call blocking probability.

Figure (15) shows the relation between the reserved cancellation probability with the number of the reserved channels for model I and model II.

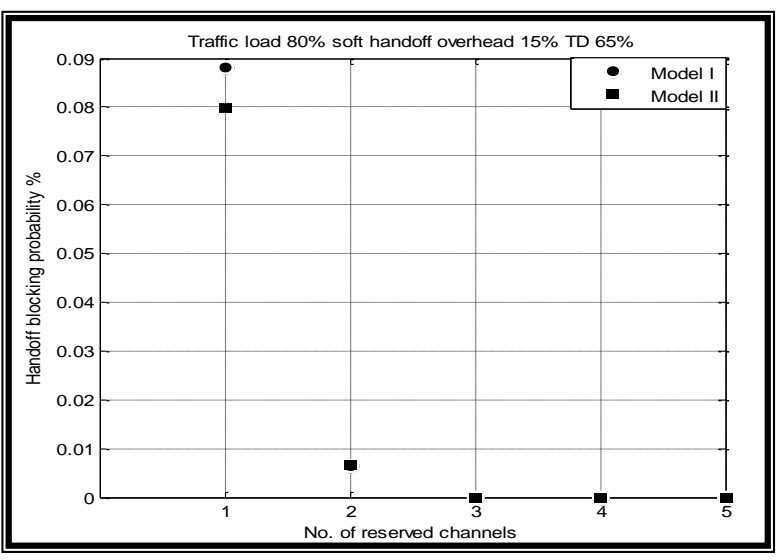

Fig. (13) Handoff blocking probability reservation pooling

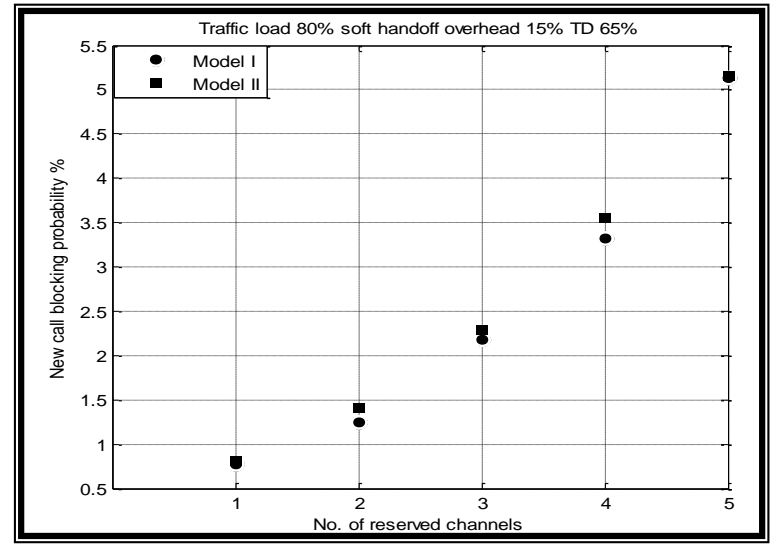

Fig. (14) New call blocking with probability with reservation pooling

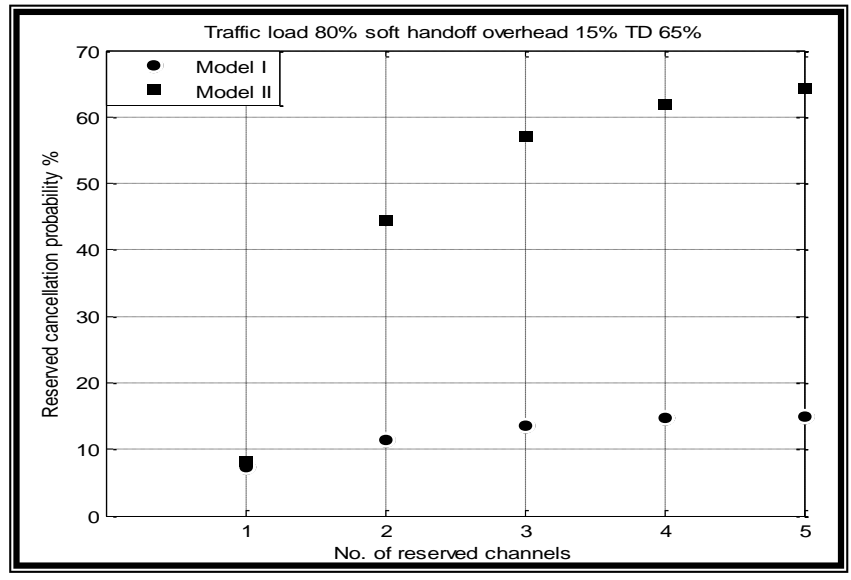

Fig. (15) Probability of reserved cancellation with reservation pooling

\section{Comparison between PCR and Guard Channels GC:}

Figures (16) and (17) show the relation between the handoff blocking probability with the number of channels (reserved and guard) for model I and model II. The handoff blocking probability is decreased with the increasing number of channels, when the reserved channels are less than the guard ones, because in PCR scheme, the channel for handoff is reserved when the MS enters the predictive reserved region and it is used for handoff when the MS arrived at the handoff region, at this time, the number of reserved free channels is increased 
and be reserved for another MS the handoff blocking probability for model II is greater than that for model I in guard channel scheme, because some of MSs ( in model II) after arriving at the handoff region and allocated a channel for handoff changed its directions which leads to an increase in the handoff blocking.

Figures (18) and (19) show the relation between the new call blocking probability with the number of channels for model I and model II. The new call blocking probability for reserved channels is less than for guard channels. The reason is that the number of calls channels equal to the total number of channels in the cell minus the number of guard channels always for the GCs. While, for PCR scheme, the number of calls channels equal to the number of channel in the cell, except at time of channel reservation, where the number call channels the total number of channels in the cell minus the number of channels reserved at this instant of time. For model II, the new call blocking probability is greater than model I. The reason for this is the changing of directions for some MSs in the handoff region.

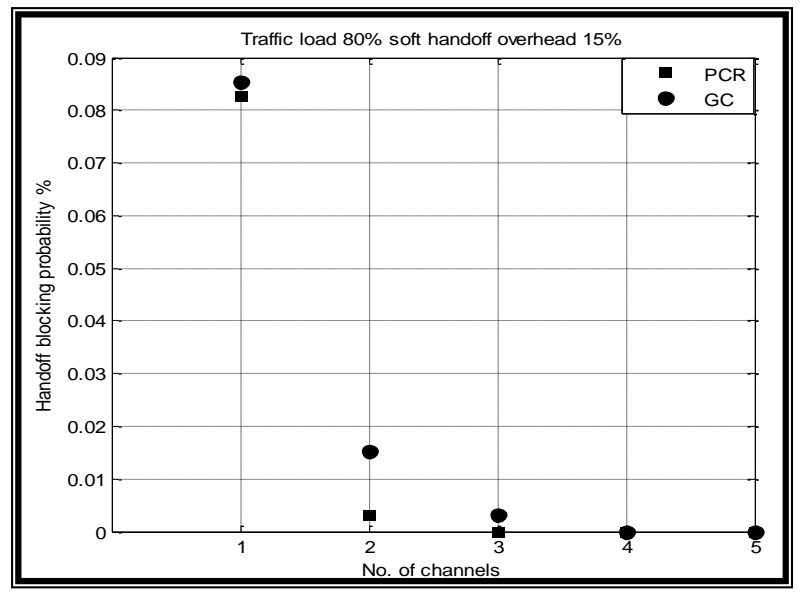

Fig. (16) Handoff blocking probability PCR and GC model I

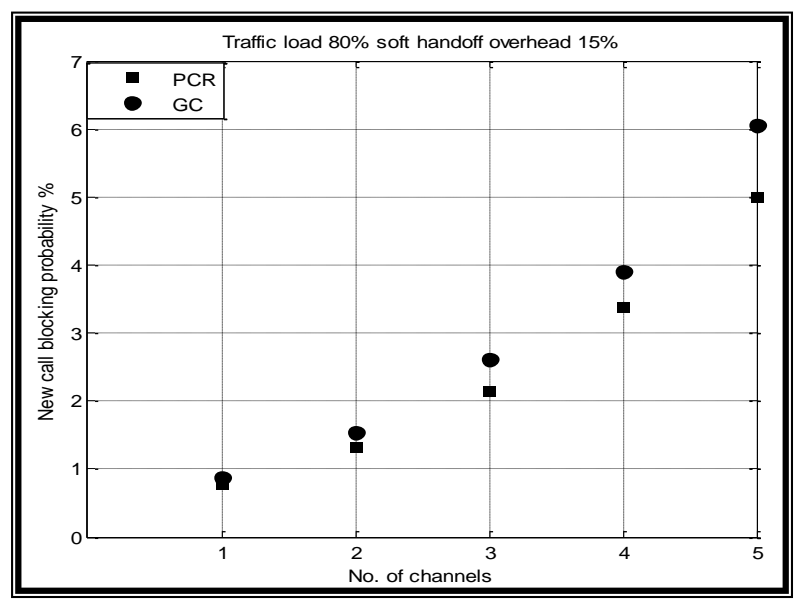

Fig. (18) New call blocking probability PCR and GC model I

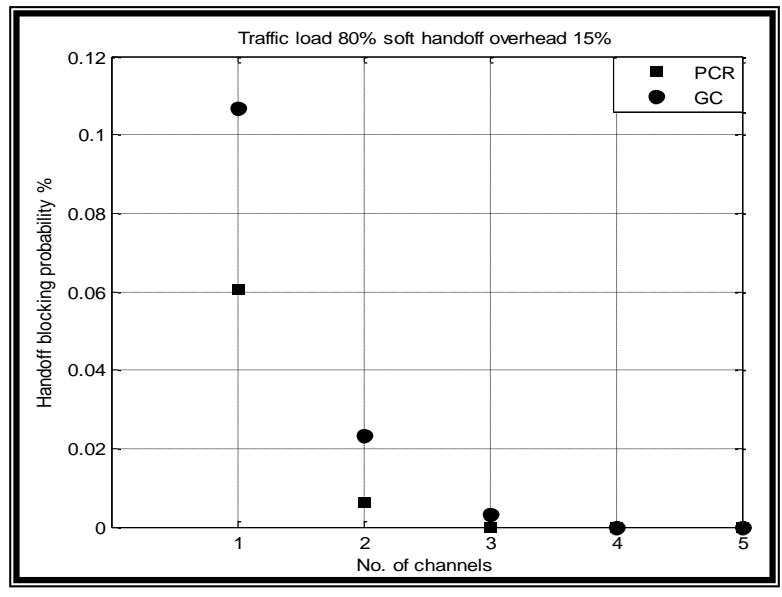

Fig. (17) Handoff blocking probability PCR and GC model II

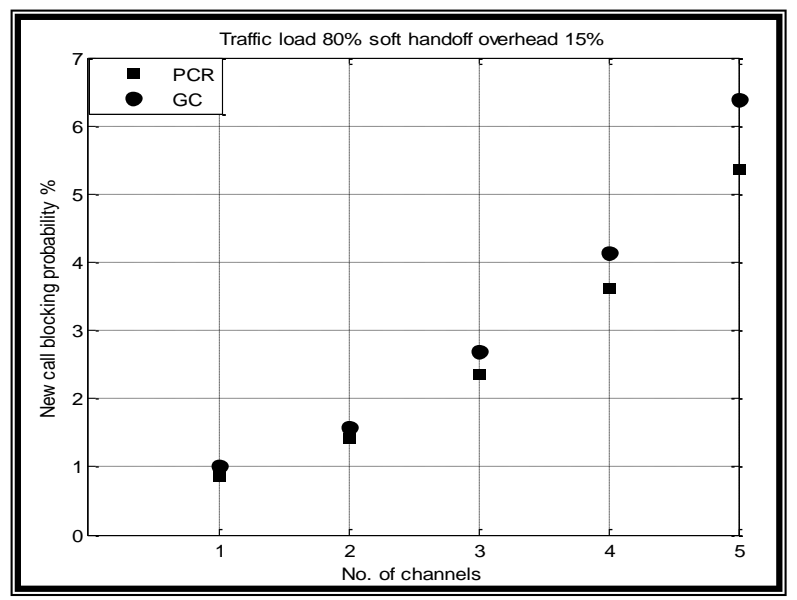

Fig. (19) New call blocking probability PCR and GC model II 


\section{Conclusions:}

The following conclusions can be drawn:

- TDOA PL technique is suitable to the estimation of the MS positioning in CDMA system, but it requires some improvements (increased power and interference cancellation) to fulfill FCC requirements.

- The interference cancellation method reduces the required power in position location measurement.

- The soft handoff blocking probability is less than the new call blocking probability due to the handoff prioritizes for the new call.

- The predictive schemes for soft handoff based on mobile positioning improve the service in mobile network.

- PCR scheme with reservation pooling method reduce the soft handoff blocking probability depending on the number of reserved channels and improve the handoff process.

- The improvement in mobile network for PCR is greater than that for guard channels.

\section{References:}

1- Y. Zhao, "Standardization of Mobile Phone Positioning for 3G Systems", IEEE Communications Magazine, July 2002.

2- Y. J. Guo, "Advances in Mobile Radio Access Networks", Artech House, 2004.

3- G. A. Mizusawa, "Performance of Hyperbolic Position Location Techniques for Code Division Multiple Access", MSc. Thesis, Virginia Polytechnic Institute and State University, August. 1996.

4- M. Aatique, "Evaluation of TDOA Techniques for Position Location in CDMA Systems", MSc. Thesis, Virginia Polytechnic Institute and State University, September. 1997.

5- M. Elkamchouchi and M. A. Mofeed, "Direction-Of-Arrival Methods (DOA) and Time Difference of Arrival (TDOA) Position Location Technique", Twenty Second National Radio Science Conference (NRSC 2005) Cairo -Egypt, March 15-17, 2005.

6- A. Lakhzouri, "Channel Estimation and Mobile Phone Positioning in CDMA Based Wireless Communication Systems", Ph.D. Thesis, Tampere University of Technology, June 2005.

7- R. I .Reza, "Data Fusion For Improved TOA/TDOA Position Determination in Wireless Systems", MSc. Thesis, Virginia Polytechnic Institute and State University, July 2000.

8- M. H. Chiu and M. A. Bassiouni, "Predictive Schemes for Handoff Prioritization in Cellular Networks Based on Mobile Positioning", IEEE, March. 2000.

9- A. K. Al-Hafith, "Radio Resource Management Using Location Estimation", MSc. Thesis, University of Mosul, 2005.

10- M. H. Chiu and M. Bassiouni, "Performance Evaluation of Position-Based Channel Reservation for Handoff of Cellular Calls", IEEE, 1999.

11- Z. Ye, L. K. Law, S. V. Krishnamurthy, Z. Xu, S. Dhirakaosal, S. K. Tripathi and M. Molle, "Predictive Channel Reservation for Handoff Prioritization in Wireless Cellular Networks", Elsevier B.V., 2006.

12- L.S. Abdulla, "The Predictive Schemes for Handoff Prioritization in Cellular Network Based on Mobile Position", M.Sc. Thesis, Mosul University, 2010.

The work was carried out at the college of Engineering. University of Mosul 